BULLETIN OF THE

AMERICAN MATHEMATICAL SOCIE'TY

Volume 77, Number 4, July 1971

\title{
GENERALIZED SOLUTIONS OF QUASILINEAR, DIFFERENTIAL INEQUALITIES. \\ I. ELLIPTIC OPERATORS
}

\author{
BY NEIL S. TRUDINGER
}

Communicated by Philip Hartman, December 28, 1970

Introduction. The purpose of this note, together with its sequel on parabolic inequalities, is to present various results describing the local and global behaviour of generalized solutions, subsolutions and supersolutions associated with quasilinear, second order differential operators of the forms

$$
\begin{gathered}
Q u=\operatorname{div} Q(x, u, D u)+B(x, u, D u), \\
P u=\operatorname{div} Q(x, t, u, D u)+\mathbb{B}(x, t, u, D u)-D_{t} u .
\end{gathered}
$$

Here $x=\left(x_{1}, \cdots, x_{n}\right)$ represents a point in a Euclidean $n$-space $E^{n} ; t$ denotes a time variable; $D u$ is the spatial gradient of the strongly differentiable function $u ; \propto$ and $B$ are respectively measurable, $n$ vector and scalar functions of their arguments and div denotes the spatial divergence.

The results announced below are a selective sampling from the work [14] and significantly extend the theory of operators $Q u, P u$ as previously developed by such authors as De Giorgi [2], Moser [5], [6], Ladyženskaya and Ural'ceva [3], [4], Serrin [1], [7], Stampacchia [8], [9], Aronson [1], Trudinger [10], [11] and others. The present note discusses the operators $Q u$, although we remark that the theory for $P u$ is largely derived through extrapolation of the $Q u$ methods. The local results for $Q u$ are in general specializations of their extensions to $P u$. The proofs of the theorems here, as supplied in [14], involve some interesting new test function techniques.

Let us restrict ourselves here to a coefficient structure determined by polynomials in $|u|$ and $|D u|$ with coefficients in $L_{p}$ spaces. For a more general type of structure see [13]. Let $\Omega$ be a bounded domain in $E^{n}$ and $G \subset \Omega \times E^{n+1}$. We define the following structural inequalities, $\mathrm{A}_{1}, \mathrm{~A}_{2}, \mathrm{~B}$, to be satisfied for all $x, u, p \in G$ :

AMS 1970 subject classifications. Primary 35B45, 35D10, 35J15, 35J60; Secondary $34 \mathrm{~J} 70$.

Key words and phrases. Generalized solution, quasilinear differential inequality, elliptic operator, parabolic operator, global estimates, maximum principle, local estimates, weak Harnack inequality, Harnack inequality, Hölder continuity, semicontinuity. 
$\mathrm{A}_{1}:|a(x, u, p)| \leqq \lambda(x) a(x)|p|^{\alpha-1}+|c(x) u|^{\alpha-1}+|f(x)|^{\alpha-1}$,

(3) $\mathrm{A}_{2}: \quad p \cdot Q(x, u, p) \geqq|\lambda(x) p|^{\alpha}-|e(x) u|^{\alpha}-|h(x)|^{\alpha}$,

$\mathrm{B}:|\beta(x, u, p)| \leqq b_{0}|\lambda(x) p|^{\alpha}+\lambda(x) b(x)|p|^{\alpha-1}$

$$
+|d(x)|^{\alpha}|u|^{\alpha-1}+|g(x)|^{\alpha},
$$

where $\alpha \geqq 1, b_{0}$ is a nonnegative constant and the coefficients $a$, $b, \cdots, h$, together with $\lambda^{-1}$ are nonnegative functions lying in various $L_{p}$ spaces. For our purposes below, we may specify here: $\lambda^{-1} \in L_{t}(\Omega)$, $a, b, c, d, e, g \in L_{s}(\Omega), f, h \in L_{r}(\Omega)$ where $r, s, t$ satisfy

$$
\frac{1}{t}+\frac{1}{s}<\frac{1}{n} \leqq \frac{1}{t}+\frac{1}{\alpha} \leqq 1, \quad \frac{1}{t}+\frac{1}{r}=\frac{1}{q} \leqq \frac{1}{\alpha} .
$$

The results in [14] are by no means confined by condition (4). For instance, in many cases the strict inequality in (4) may be weakened and the lower bound on $n$ implied there is not necessary.

In terms of the structure, we may define a convenient notion of solution (for a more natural and general notion see $[14]$ ). Let $V^{0}(\Omega)$ denote the completion of $C_{0}^{1}(\Omega)$ under the $L_{\alpha}(\Omega)$ norm of $|D u|$ with respect to the measure $d \mu=\lambda^{\alpha} d x$ and let

$$
V^{\mathrm{loc}}(\Omega)=\left\{u ; \phi u \in V^{0}(\Omega) \text { for all } \phi \in C_{0}^{1}(\Omega)\right\} .
$$

Set

$$
Q(u, \phi)=\int_{\Omega}\{D \phi \cdot Q(x, u, D u)-\phi \beta(x, u, D u)\} d x .
$$

A function, $u \in V^{\mathrm{loc}}(\Omega)$, satisfies $Q u \geqq 0(Q u \leqq 0)$ in $\Omega$ if $-\infty<Q(u, \phi)$ $\leqq 0(\infty>Q(u, \phi) \geqq 0)$ for all $\phi \geqq 0, \in V^{0}(\Omega)$. Furthermore, $u$ is said to satisfy $u \leqq L$ on $\partial \Omega$, in the sense of $V^{0}(\Omega)$, if the function $v$ $=\sup (u-L, 0) \in V^{0}(\Omega)$.

Global estimates. Assume now, that conditions $\mathrm{A}_{2}$ and $\mathrm{B}$ hold on $G=\Omega \times E^{n+1}$ with $b_{0}=0$. Then we have

Theorem 1. Let $u \in V^{\mathrm{loo}}(\Omega)$ satisfy $Q u \geqq 0$ in $\Omega$ and $u \leqq L<\infty$ on $\partial \Omega$. Then setting $v=\sup (u-L, 0)$ we have

(i) if $q>n, v$ is in $L_{\infty}(\Omega)$;

(ii) if $q=n, v$ is in the Orlicz space $L_{\phi}(\Omega)$ where $\phi=e^{|t|}-1$;

(iii) if $q<n$, $v$ is in $L_{p}(\Omega)$ where $1 / p=1 / q-1 / n$.

In each case the norm of $v$ may be estimated in terms of $L, n, r, s, t, \alpha$, $\|u\|_{L_{\alpha}(\Omega)},|\Omega|$ and the relevant norms of $\lambda^{-1}, a, \cdots, h$. 
To obtain an $a$ priori estimate for sup $u$, independent of $\|u\|_{L_{\alpha}(\Omega)}$, we assume, additionally to case (i) above, that $d=e=0$.

Theorem 2. Let $u \in V^{\text {loc }}(\Omega)$ satisfy $Q u \geqq 0$ in $\Omega$. Then

$$
\sup _{\Omega} u \leqq \sup _{\partial \Omega} u+C\left\{\|g\|^{\alpha /(\alpha-1)}+\|h\|\right\}
$$

where the constant $C$ depends on $n, r, s, t,|\Omega|,\left\|\lambda^{-1}\right\|,\|b\|$ and $g=0$ if $\alpha=1$.

Corresponding results for supersolutions and consequently solutions follow automatically. Note also that if $|\beta(x, u, p)|$ is replaced by $\operatorname{sign} u \cdot B(x, u, p)$ in condition $\mathrm{B}$, then Theorem 2 holds for the function $u^{+}=\sup (u, 0)$.

Local estimates. A local version of Theorem 1 is true if $A_{1}, A_{2}$ and $B$ all hold on $G=\Omega \times E^{n+1}$, with $b_{0}=0$. See [14] for the detailed statement. Let us instead consider bounded solutions and assume that conditions $\mathrm{A}_{1}, \mathrm{~A}_{2}$ and $\mathrm{B}$ hold on sets $G_{M}=\Omega \times(-M, M) \times E^{n}$ with $r=s, \alpha>1$. For supersolutions the following estimate, which reduces to a weak Harnack inequality when $f=g=h=0$, is derived.

THEOREM 3. Let $u$ be a bounded, nonnegative function in $V^{\mathrm{loc}}(\Omega)$ satisfying $Q u \leqq 0$ in $\Omega$ and let $B$ be a ball in $\Omega$. Then for any smaller concentric balls, $B_{1}$ and $B_{2}$, we have

$$
\left(\left|B_{1}\right|^{-1} \int_{B_{1}} u^{\alpha-1}\right)^{1 /(\alpha-1)} \leqq C\left(\inf _{B_{2}} u+k\right)
$$

where the constant $C$ depends on $n, s, t, b_{0} \sup _{B} u,|B|,\left|B_{1}\right|,\left|B_{2}\right|$ and the norms of $\lambda^{-1}, a, b, \cdots$, e over $B$ while $k=|B| 1 / n-1 / q\left(\|f\|_{B}+\|h\|_{B}\right)$ $+\left(|B|^{1 / n-1 / q}\|g\|_{B}\right)^{\alpha /(\alpha-1)}$.

Some important corollaries of Theorem 3 are:

I. a strong maximum principle for subsolutions of $Q$ when a homogeneous structure prevails,

I1. a Harnack inequality for locally bounded solutions,

III. a Hölder estimate for locally bounded solutions when an additional coefficient restriction, namely that

$$
\Lambda(B)=|B|^{-(1 / t+1 / s)}\left\|\lambda^{-1}\right\| B\|a\|_{B}
$$

is bounded uniformly on balls $B \subset \Omega$, is satisfied,

IV. the semicontinuity of subsolutions and supersolutions, also under the above restriction. This result also extends to the case $\alpha=1$ and consequently embraces the minimal surface equation. 
Finally, we note that the theorems above are new even in the nondegenerate case, $t=\infty$, and also for linear elliptic operators of the form

$$
L u=\frac{\partial}{\partial x_{i}}\left(a^{i j}(x) u_{x_{j}}+a^{i}(x) u+f^{i}\right)+b^{i}(x) u_{x_{j}}+a(x) u+f,
$$

ellipticity meaning that the matrix $\left[a^{i j}(x)\right]$ is positive almost everywhere in $\Omega$. New existence and uniqueness theorems for $L u$ follow from Theorem 2 .

\section{REFERENCES}

1. D. G. Aronson and J. Serrin, Local behavior of solutions of quasilinear parabolic equations, Arch. Rational Mech. Anal. 25 (1967), 81-122. MR 39 \#5952.

2. E. De Giorgi, Sulla differenziabilità e l'analiticità delle estremali degli integrali multipli regolari, Mem. Accad. Sci. Torino. Cl. Sci. Fis. Mat. Nat. (3) 3 (1957), 25-43. MR $20 \# 172$.

3. O. A. Ladyženskaja and N. N. Ural'ceva, Linear and quasilinear equations of elliptic type, "Nauka", Moscow, 1964; English transl., Academic Press, New York, 1968. MR $35 \# 1955$; MR 39 \#5941.

4. O. A. Ladyženskaja, V. A. Solonnikov and N. N. Ural'ceva, Linear and quasilinear parabolic equations, "Nauka", Moscow, 1967; English transl., Transl. Math. Monographs, vol. 23, Amer. Math. Soc., Providence, R. I., 1968. MR 39 \#3159a, b.

5. J. Moser, On Harnack's theorem for elliptic differential equations, Comm. Pure Appl. Math. 14 (1961), 577-591. MR 28 \#2356.

6. ——, A Harnack inequality for parabolic differential equations, Comm. Pure Appl. Math. 17 (1964), 101-134. MR 28 \#2357.

7. J. Serrin, Local behaviour of solutions of quasi-linear equations, Acta Math. 111 (1964), 247-302. MR $30 \# 337$.

8. G. Stampacchia, Contributi alla regolarizzazione delle soluzioni dei problemi al contorno per equasione del secondo ordine ellittiche, Ann. Scuola Norm. Sup. Pisa (3) 12 (1958), 223-245. MR 23 \#A2616.

9. - Le problème de Dirichlet pour les équations elliptiques du second ordre d coefficients discontinus, Ann. Inst. Fourier (Grenoble) 15 (1965), fasc. 1, 189-258. MR 33 \#404.

10. N. S. Trudinger, On Harnack type inequalities and their application to quasilinear elliptic equations, Comm. Pure Appl. Math. 20 (1967), 721-747. MR 37 \#1788.

11. - Pointwise estimates and quasilinear parabolic equations, Comm. Pure Appl. Math. 21 (1968), 205-226. MR 37 \#1758.

12. - On the regularity of generalized solutions of linear, nonuniformly elliptic equations, Arch. Rational Mech. Anal. 41 (1971) (to appear).

13. - Continuity of weak solutions of quasilinear equations, Indiana Univ. Math. J. (to appear).

14. - Local and global behaviour of generalized solutions of quasilinear, differential inequalities (in preparation).

University of Minnesota, Minneapolis, Minnesota 55455

University of Queensland, St. Lucia, QueEnsland 4067, Australia 